\title{
Article
}

\section{Characterisation of the mouse diabetes susceptibility locus Nidd/SJL: islet cell destruction, interaction with the obesity QTL Nob1, and effect of dietary fat}

\author{
L. Plum² ${ }^{2}$ K. Giesen ${ }^{2}$, R. Kluge ${ }^{3}$, E. Junger ${ }^{4}$, K. Linnartz² ${ }^{2}$ A. Schürmann², W. Becker ${ }^{2}$, H.-G. Joost ${ }^{1,2}$ \\ ${ }^{1}$ German Institute of Human Nutrition, Potsdam-Rehbrücke, Germany \\ ${ }^{2}$ Institute of Pharmacology and Toxicology, ${ }^{3}$ Institute of Animal Research, Medical Faculty, Technical University of Aachen, \\ Aachen, Germany \\ ${ }^{4}$ German Institute of Diabetes Research, Düsseldorf, Germany
}

\section{Abstract}

Aims/hypothesis. The diabetes susceptibility locus Nidd/SJL was identified in an outcross of New Zealand obese (NZO) and lean Swiss/Jackson Laboratory mouse strain (SJL) mice. Here we characterise its effects in a $\mathrm{NZO} \times \mathrm{F} 1(\mathrm{SJL} \times \mathrm{NZO})$ backcross population raised on high-fat or standard diet, and describe its interaction with the obesity quantitative trait locus (QTL) Nobl.

Methods. NZO $\times$ F1 $(\mathrm{SJL} \times \mathrm{NZO})$ backcross mice were raised on a normal or high fat diet and were monitored (body weight, blood glucose, serum insulin) for 22 weeks. Genotypes of polymorphic markers were determined by PCR, and linkage analysis was done. Pancreas morphology was assessed by conventional staining and immunohistochemistry of insulin.

Results. In backcross mice raised on a high-fat diet, Nidd/SJL produced hyperglycaemia (maximum likelihood of the odds (LOD) score 9.9), hypoinsulinaemia, reduction of islet-cell volume, and loss of beta cells. No effect was observed on body weight and serum in- sulin concentrations before the onset of hyperglycaemia. The development of diabetes in carriers of Nidd/SJL was markedly accelerated and aggravated by the obesity/hyperinsulinaemia QTL Nob1; together, these loci were responsible for approximately $90 \%$ of the diabetes observed in the backcross population. When raised on a standard diet, Nidd/SJL carriers exhibited a fivefold higher prevalence of diabetes, but Nobl failed to enhance the effect of Nidd/SJL.

Conclusion/interpretation. Diabetes in this obese mouse model is the result of an interaction of genes responsible for obesity/insulin resistance (e.g. Nobl) and islet cell failure (Nidd/SJL). The combined diabetogenic effects of Nidd/SJL and Nobl were markedly enhanced by a high-fat diet, whereas that of Nidd/SJL alone was independent of the dietary fat content. [Diabetologia (2002) 45:823-830]

Keywords Obesity, insulin resistance, New Zealand obese mouse, quantitative trait locus, dietary fat, leptin receptor.
New Zealand obese (NZO) mice have a polygenic syndrome of hyperphagia, obesity and insulin resis-

Received: 30 November 2001 / Revised: 23 January 2002

Published online: 26 April 2002

(C) Springer-Verlag 2002

Corresponding author: H.-G. Joost, Institut für Pharmakologie und Toxikologie, Medizinische Fakultät der RWTH Aachen, Wendlingweg 2, D-52057 Aachen, Germany, e-mail: joost @ rwth-aachen.de

Abbreviations: QTL, Quantitative trait locus; LOD, likelihood of the odds; NOD, non-obese diabetic mouse strain; NON, non-obese non-diabetic mouse strain; NZO, New Zealand obese mice; SJL, Swiss/Jackson Laboratory mouse strain tance [1] that resembles the human metabolic syndrome [2]. The strain has been used to characterise the interaction of obesity and diabetes genes ('diabesity'), and to identify diabetes genes from other mouse strains that accelerate the development of hyperglycaemia in NZO [3]. We have previously established a backcross model of NZO mice with the lean and normoglycaemic Swiss/Jackson Laboratory mouse (SJL) strain in order to identify and locate susceptibility loci (QTL) for obesity, insulin resistance, and diabetes [4, 5]. With this model, we localised a QTL (Nidd/SJL) for severe hyperglycaemia on chromosome 4 that was contributed by the lean SJL strain. Nidd/SJL appeared to enhance the effects of obesity genes on the develop- 
ment of severe hyperglycaemia in the NZO mouse, and was responsible for $60-90 \%$ of the diabetes prevalence in the backcross population.

Hyperglycaemia in obese mouse models is believed to reflect the insufficiency of the endocrine pancreas to compensate for insulin resistance. The molecular link between insulin resistance and islet cell degeneration, however, is not clear. Because the development of obesity-associated diabetes ('diabesity') is markedly dependent on the specific genetic background, and is enhanced by the introduction of diabetes genes [3, $4,6]$, a specific mechanism injuring the beta cell has to be considered. Candidates that might mediate betacell destruction are the serum concentrations of glucose ('glucose toxicity') or fatty acids ('lipotoxicity') which are both higher during the period of insulin resistance and impaired glucose tolerance. At present, there is only circumstantial evidence favouring these hypotheses. However, it appears reasonable to assume that the characterisation of susceptibility loci, and the identification of the responsible genes will ultimately elucidate the mechanisms that are responsible for islet-cell failure in Type II diabetes mellitus.

In this study, we characterised the effects of the diabetogenic allele of the Nidd/SJL locus with regard to its effects on insulin concentrations, on islet morphology, and on the time course of development of hyperglycaemia. In addition, we defined its interaction with the obesity QTL Nob1, and studied the effects of dietary fat content on the diabetogenic effect of Nidd/SJL alone and in combination with Nobl in the NZO $\times$ SZO backcross population. The data indicate that diabetes in this obese mouse model is the result of interacting genes responsible for obesity/insulin resistance (e.g. Nobl) and islet cell failure $(N i d d / S J L)$. Furthermore, the combined diabetogenic effects of Nidd/SJL and Nobl were markedly enhanced by a high-fat diet, whereas that of Nidd/SJL alone was independent of the dietary fat content.

\section{Methods and Materials}

Mice. SJL (SJL/NBom) and NZO mice (NZO/HIBom) were obtained from Bomholtgard (Ry, Denmark). Female SJL and male NZO mice were used to found an F1 generation, and backcrosses $(\mathrm{SJL} \times \mathrm{F} 1$ and $\mathrm{NZO} \times \mathrm{F} 1)$ were carried out. After weaning (3 weeks of age), mice received standard (Altromin, Lage, Germany; no. 1314, with 5, 48, and $22.5 \%$ fat, carbohydrates, and protein, respectively; $12.5 \mathrm{~kJ} / \mathrm{g}$ metabolising energy) or high fat (no. C1057, with 16.0, 46.8, 17.1\% fat, carbohydrates, and protein, respectively; $15.4 \mathrm{~kJ} / \mathrm{g}$ ) rodent chow. Throughout the study, mice had free access to food and water. Three to six mice were kept in each cage (Macrolon, type III) in a temperature controlled room $\left(20^{\circ} \mathrm{C}, 55 \pm 5 \%\right.$ relative humidity) with a 12-h light-dark cycle and lights on at 06:00 h. Mice were killed at the age of 22 weeks in isoflurane anaesthesia by exsanguination. The principles of laboratory animal care (NIH publication no. 85-23) were followed, and the study was approved by the committee for ethics of animal experimentation at the district administration in Köln, Germany.
Body weight and BMI. Body weight and food consumption were determined once every week during the study period. Body length (without tail) was measured once after 22 weeks with the millimetre scale of plotting paper on which the carcasses were fixated. The BMI was calculated as an additional measure of obesity $(\mathrm{BMI}=$ body weight $[\mathrm{g}] /($ body length $\left.[\mathrm{cm}])^{2}\right)$.

Serum parameters. Blood glucose was determined with the glucometer Elite (Bayer, Leverkusen, Germany). Serum (week 10) and plasma insulin (week 22) was determined by radioimmunoassay (Amersham-Pharmacia, Freiburg, Germany) with anti-rat insulin antiserum and ${ }^{125}$ I-labelled rat insulin as tracer. Free and bound radioactivity were separated with an anti-IgG antibody.

Genotyping, genetic maps, and QTL analysis. DNA was prepared from mouse tails with a DNA isolation kit (InViTek, Berlin, Germany) based on a salt precipitation method. Animals were genotyped for polymorphic microsatellite markers by PCR with oligonucleotide primers obtained from Research Genetics (Huntsville, Al., USA), and microsatellite length was determined by non-denaturing polyacrylamide gel electrophoresis (10\% gels). A quantitative trait locus (QTL) analysis was done as described with the programme Mapmaker/QTL 1.1 [7] after construction of the genetic map with Mapmaker/EXP 3.0 [8]. Mice heterozygous for the markers D4Mit278 and D4Mit204 were considered carriers of the diabetogenic allele of the locus Nidd/SJL. The genotype of the obesity/insulin resistance locus Nobl was determined with the marker D5Mit392 (homozygosity).

Histology of the pancreas. Pancreatic tissue was excised immediately after exsanguination at week 22, fixed in paraformaldehyde (4\% solution in phosphate buffered saline) for $10 \mathrm{~h}$, and embedded in paraffin according to standard procedures. Serial sections $(5 \mu \mathrm{m}$, at sampling intervals of $300 \mu \mathrm{m})$ were cut in parallel to the longitudinal axis of the gland. Sections were immunostained with guinea pig anti-porcine insulin antibody (DAKO Diagnostika, Hamburg, Germany) and peroxidase-linked secondary antibody, and were counterstained with haematoxylin. The relative volume of islets was estimated by light microscopic point counting. At least four serial sections were evaluated for each pancreas. Apoptotic cells were detected in paraffin sections of mouse pancreas with the aid of the TdT-FragEL DNA Fragmentation Detection Kit (Oncogene Research Products, Boston, Mass., USA) according to manufacturer's protocol.

\section{Results}

The development of diabetes in the NZO $\times$ SZO backcross population and early hyperinsulinaemia and obesity. In order to characterize the development of diabetes in theNZO $\times$ SJL backcross model, a total of 199 male $\mathrm{NZO} \times \mathrm{F} 1(\mathrm{SJL} \times \mathrm{NZO})$ mice were generated and raised on a high-fat diet. At or before week 17, 61 backcross mice $(30.5 \%)$ exhibited severe, decompensated hyperglycaemia (blood glucose $>16 \mathrm{mmol} / \mathrm{l}$ ), at week 22, 88 mice (44\%) were diabetic. Body weights of diabetic and non-diabetic mice were essentially identical at week $4(13.6 \pm 0.2$ vs. $13.1 \pm 0.2 \mathrm{~g}$, means \pm SEM). Thereafter, mice that later became diabetic ex- 


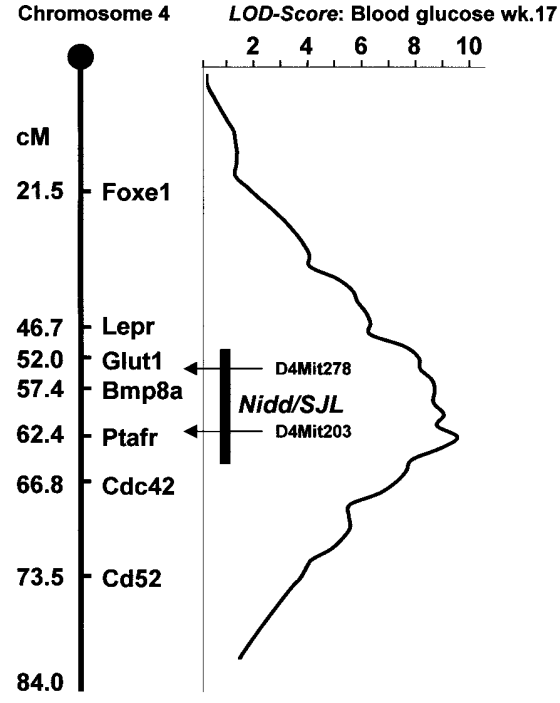

Fig. 1. Localisation of the susceptibility locus (Nidd/SJL) for diabetes in the $\mathrm{NZO} \times \mathrm{SZO}$ backcross population on chromosome 4. A genetic map of the chromosome was generated for the genotyped markers with the MAPMAKER/EXP programme, and LOD scores were subsequently calculated (MAPMAKER/QTL) with data from 199 male NZO $\times$ F1 $($ SJL $\times$ NZO) backcross mice. Positions of the known genes shown in the figure are given in $\mathrm{cM}$ as obtained from a linkage map of the Mouse Genome Informatics database (http://www.informatics.jax.org)

hibited higher body weights than the non-diabetic animals, the difference being $4.3 \mathrm{~g}$ at week $10(48.2 \pm 0.5$ vs. $43.9 \pm 0.5 \mathrm{~g})$, and $5.7 \mathrm{~g}$ at week $14(56.4 \pm 0.6$ vs. $50.7 \pm 0.6 \mathrm{~g}$ ). At week 10, diabetic animals exhibited markedly higher insulin concentrations than non-diabetic mice $(13.1 \pm 1.8$ vs $5.4 \pm 0.6 \mathrm{ng} / \mathrm{ml})$. In contrast, insulin concentrations at week 22 were comparable in both groups $(22.3 \pm 3.2 \mathrm{vs} 24.6 \pm 3.3 \mathrm{ng} / \mathrm{ml})$, reflecting the failure of the endocrine pancreas to compensate for the severe hyperglycaemia $(26.8 \pm 1.0$ vs $10.1 \pm$ $0.3 \mathrm{mmol} / \mathrm{l}$ in the non-diabetic mice). Thus, early hyperinsulinaemia and obesity appeared to be predictors of diabetes in the backcross population.

The diabetes susceptibility locus Nidd/SJL produces hypoinsulinaemia and beta-cell loss but not obesity and early hyperinsulinaemia. Consistent with our previously published results [4], heterozygosity for the marker D4Mit278 predicted diabetes in the backcross population at or before week 22 (frequencies of the Nidd/SJL allele $28.6 \%$ in the non-diabetic, and $54.5 \%$ in the diabetic population). The distribution of LOD scores on chromosome 4 showed a very broad region of highly significant LOD scores with maximum values between the markers D4Mit278 and D4Mit203, suggesting that the observed effect might be conferred by more than one variant gene present in this locus (Fig. 1). Therefore, only animals heterozygous for both markers were considered carriers of the diabetogenic allele Nidd/SJL in the following analysis (77 an-
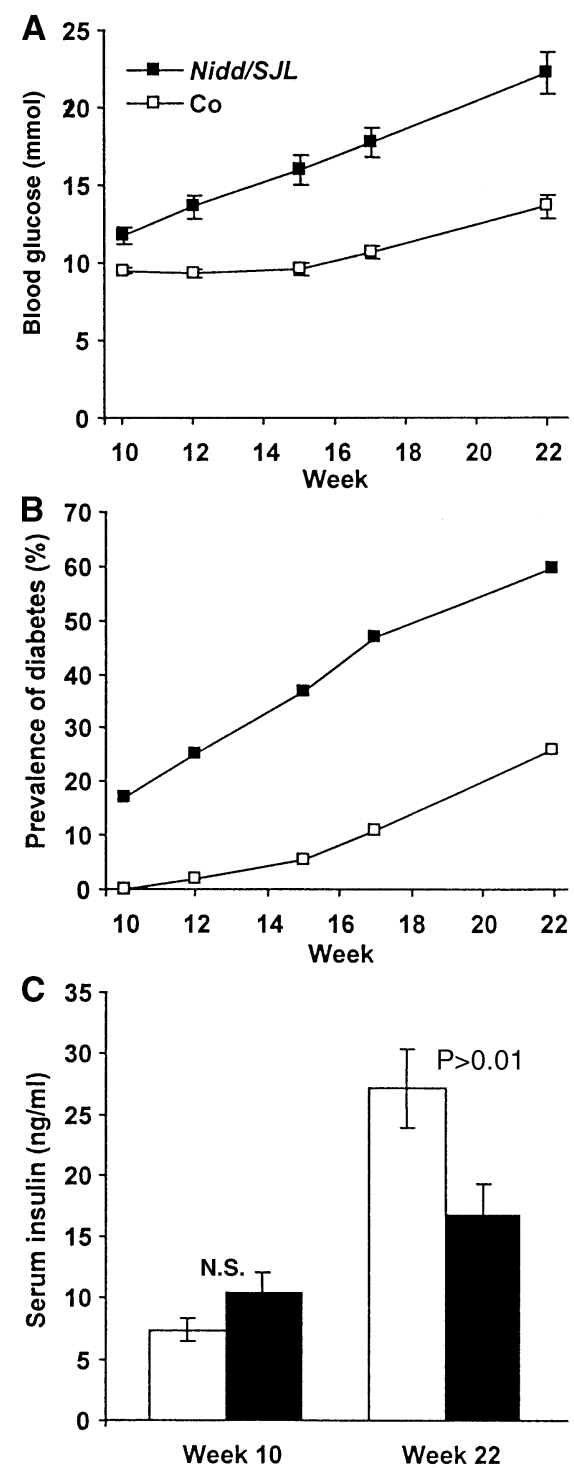

Fig. 2A-C. Hyperglycaemia and hypoinsulinaemia in carriers of the diabetes susceptibility locus $N i d d / S J L$ in the $\mathrm{NZO} \times \mathrm{F} 1$ backcross population. Mice heterozygous (Nidd/SJL carriers, $n=77$, filled symbols and bars) or homozygous (Co, $n=108$, open symbols and bars) for both markers D4Mit278 and D4Mit 203 were monitored for 22 weeks. A Time course of the development of hyperglycaemia. Differences were significant at $p<0.001$ (week 10) or $p<10^{-5}$ (week 12-22). B Time course of the prevalence of diabetes (\% of animals with hyperglycaemia $>16 \mathrm{mmol} / \mathrm{l})$; $\mathbf{C}$ serum insulin concentrations at weeks 10 and 22

imals were heterozygous for both markers, 108 were homozygous, and 14 presented recombinations between the markers).

Figure 2 illustrates the effect of $N i d d / S J L$ on the development of hyperglycaemia. The presence of the diabetogenic allele was associated with a marked increase in the prevalence of diabetes (hyperglycaemia $>16 \mathrm{mmol} / \mathrm{l})$. It should be noted that diabetes also developed in mice lacking the diabetogenic allele (homozygotes), but started much later. None of these mice became hyperglycaemic before the age of 15 

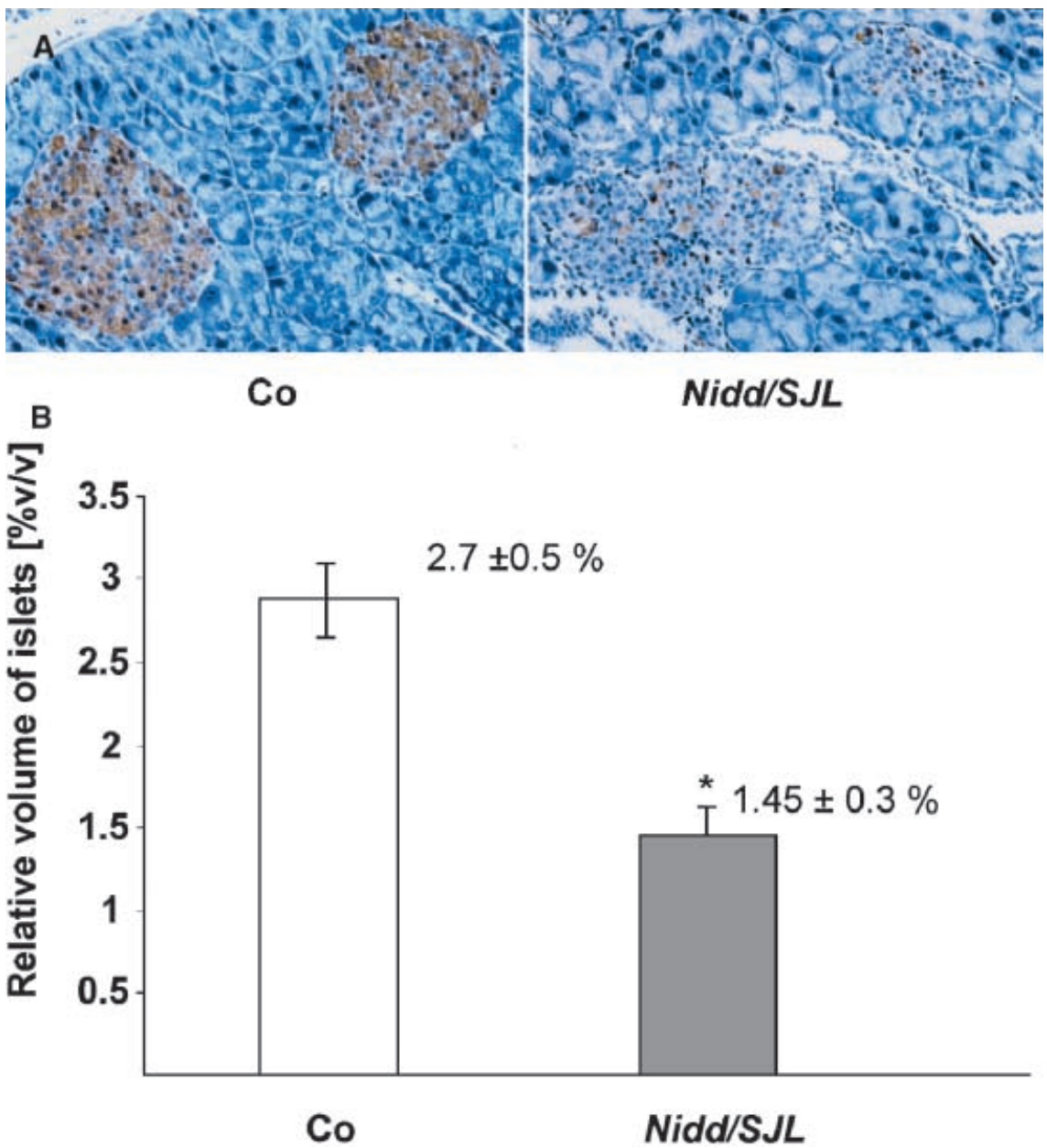

Fig. 3A, B. Effect of $N i d d / S J L$ on islet morphology and relative islet volume in $\mathrm{NZO} \times \mathrm{F} 1(\mathrm{SJL} \times \mathrm{NZO})$ backcross mice. Pancreas was isolated from male backcross mice, fixed, stained with hematoxylin, and immuno-stained for insulin as described under Materials and Methods. A Islet morphology of representative samples of pancreatic sections from a control mouse and a Nidd/SJL carrier. B In $11 \mathrm{Nidd} / S J L$ carriers and 11 control mice, islet volume was determined and expressed as $\%$ of the total volume of pancreatic tissue (means \pm SEM). The difference was significant at $p<0.05$

weeks, whereas a considerable portion of the Nidd/SJL carriers exhibited diabetes before this time point. Thus, Nidd/SJL seemed to enhance and accelerate the effect of other diabetogenic alleles contributed by the NZO genome. Confirming our previous results [4], we found that insulin concentrations were markedly lower in Nidd/SJL mice than in the control mice (mice homozygous for the NZO allele of D4Mit278) at week 22. At week 10, in contrast, insulin concentrations appeared similar in both groups, indicating that Nidd/SJL did not produce early hyperinsulinaemia. There was no difference in the body weights between carriers of the diabetogenic allele and non-carriers until week 14 (week $10,45.5 \pm 0.5 \mathrm{~g}$ in controls vs $46.1 \pm 0.6$ in $N i d d / S J L$; week $12,49.2 \pm 0.6 \mathrm{~g}$ vs
$49.7 \pm 0.7$; week $14,52.8 \pm 0.7 \mathrm{~g}$ vs $53.1 \pm 0.7)$. Thereafter, as was described previously [4], body weights in the Nidd/SJL group were lower, presumably because of a diabetes-induced weight loss (week 22, $63.8 \pm 1.0 \mathrm{~g}$ in controls vs. $59.7 \pm 0.9 \mathrm{in} \mathrm{Nidd} / \mathrm{SJL}$ ).

Because most diabetic animals carrying the Nidd/SJL allele had severe hypoinsulinaemia, we concluded that the diabetogenic effect reflected a loss of insulin-producing beta cells. As is illustrated in Figure 3 (lower panel), the relative volume of islets was significantly reduced in carriers of the Nidd/SJL locus, in parallel with the reduction in serum insulin (Fig. 2C). Furthermore, islets of diabetic animals carrying Nidd/SJL had severe morphological alterations (Fig. 3, upper panel) including degranulation of beta cells, intrusion of exocrine cells, and apoptotic islet cells (TUNEL positive, data not shown). Leucocytic infiltrates were frequently seen along the ducts and at peri-insular locations. In contrast to the backcross population, no morphological anomalies could be detected in parental SJL mice or in the F1 progeny (data not shown).

Interaction of Nidd/SJL with the obesity locus Nob1. Because only 58 of a total of 87 diabetic animals carried the diabetogenic Nidd/SJL allele, we assumed that other susceptibility loci also contributed to 

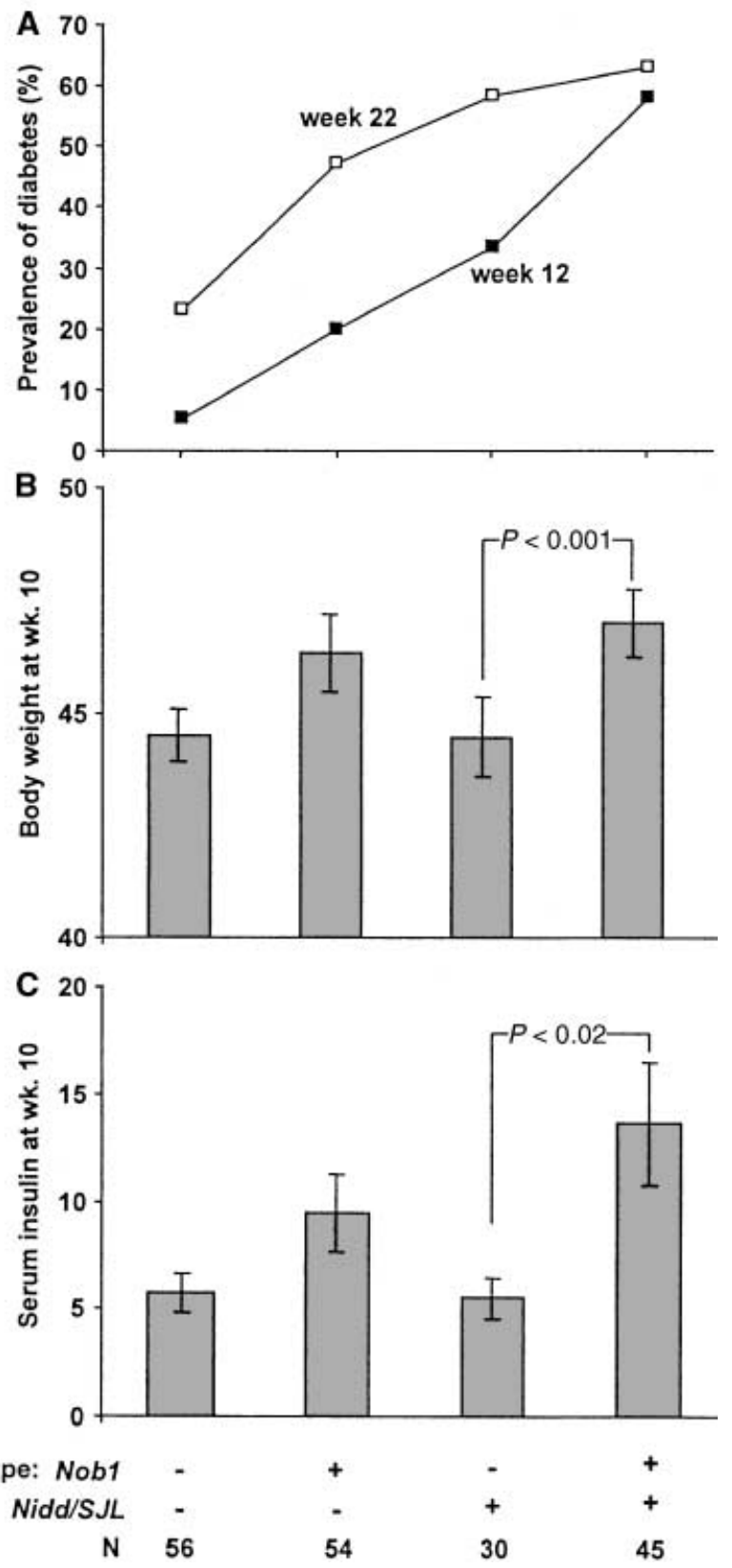

Fig. 4A-C. Interaction of $N i d d / S J L$ with the obesity QTL Nobl in NZO $\times$ F1 $(\mathrm{SJL} \times \mathrm{NZO})$ backcross mice at a high fat diet. Data from 185 male backcross mice were analysed with regard to their genotype at Nidd/SJL (heterozygous at D4Mit278 and D4Mit203) and Nobl (homozygous at D5Mit392). The number of animals in each group $(n)$ is given below the genotype. A Prevalence of severe hyperglycaemia $(>16 \mathrm{mmol} / \mathrm{l})$ at week 17 (filled squares) and week 22 (open squares); B body weight at week 10 as a parameter of early obesity; $\mathbf{C}$ serum insulin at week 10 as a parameter of early insulin resistance; means \pm SEM

the diabetes in the backcross. Thus, we conducted an additional genome wide search for diabetes loci which identified a QTL for hyperglycaemia on chromosome 5 near the marker D5Mit392 (maximum LOD-score 3.1) which maps $1 \mathrm{cM}$ distal to a previously described QTL for hyperglycaemia (unnamed, maximum LOD
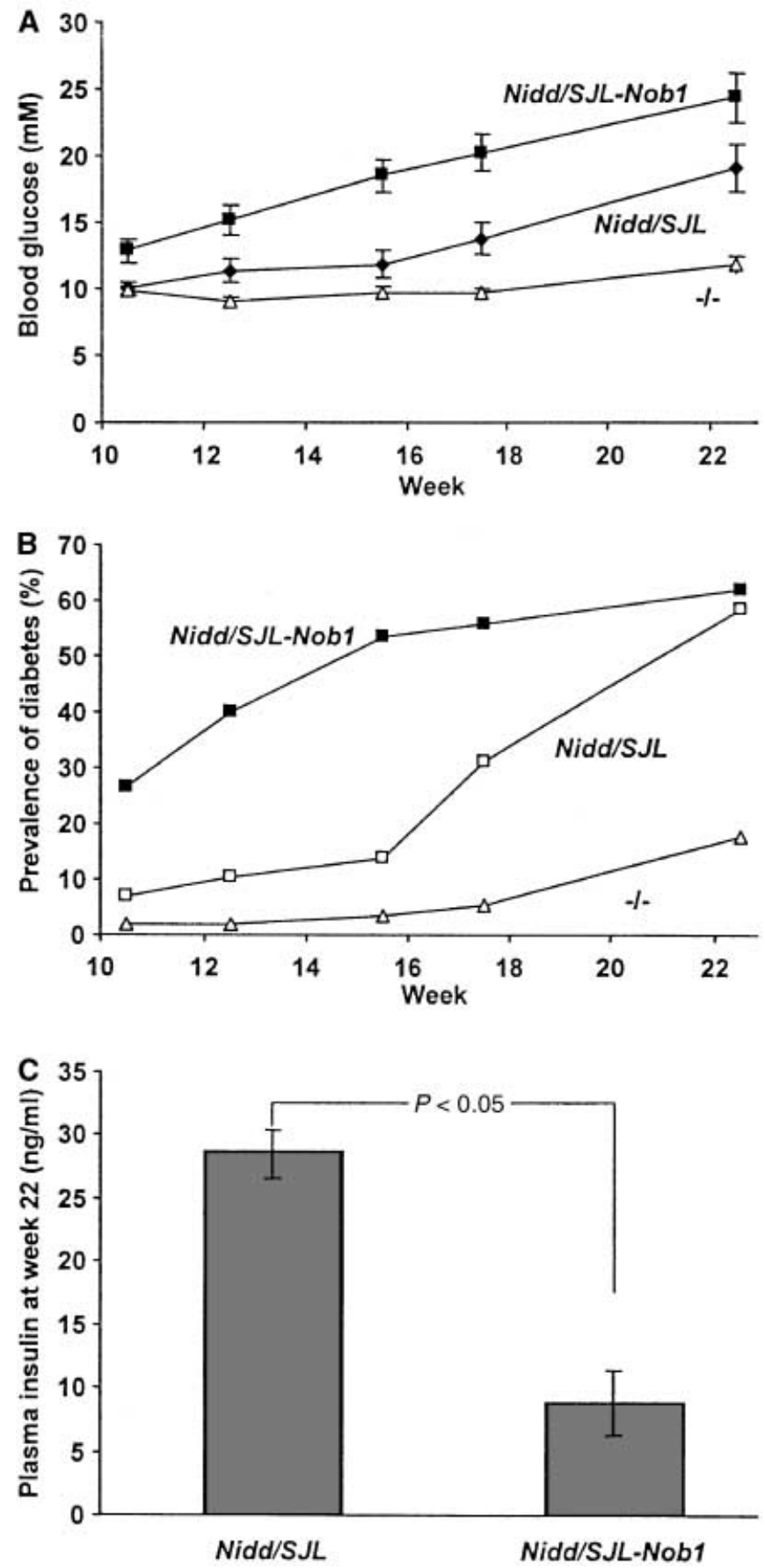

Fig. 5A-C. Nobl accelerates the development of diabetes and aggravates its manifestation in carriers of the diabetogenic Nidd/SJL allele - The time course of the development of hyperglycaemia was analysed in male $\mathrm{NZO} \times \mathrm{F} 1(\mathrm{SJL} \times \mathrm{NZO})$ backcross mice separated according to their genotype at Nidd/SJL and Nobl ( $n=45$ with both Nidd/SJL and Nob1; $n=30$ with Nidd/SJL only; (-/-), $n=56$ non-carriers of both diabetogenic alleles). A Time course of the prevalence of severe hyperglycaemia (>16 mmol/l); $\mathbf{B}$ time course of the development of hyperglycaemia; $\mathbf{C}$ plasma insulin levels at week 22 in diabetic mice carrying one (Nidd/SJL, $n=17)$ or both $(N i d d / S J L$ Nob1, $n=24)$ diabetogenic alleles 
score at D5Mit81) [3]. This QTL appears to be identical with the obesity locus Nobl which we have previously identified in a female NZO $\times$ F1 $($ SJL $\times N Z O)$ backcross population [5]. As is illustrated in Figure 4, Nobl increased the prevalence of diabetes, and markedly enhanced the effect of Nidd/SJL. At week 17 or $22,93 \%$ or $89 \%$, respectively, of the diabetic mice carried the diabetogenic Nidd/SJL, or two Nobl alleles, or both. In contrast to Nidd/SJL, Nobl increased body weight and serum insulin concentrations before the onset of diabetes (week 10). Interestingly, the combination of Nidd/SJL with Nob1 accelerated the development of hyperglycaemia and aggravated its manifestation (Fig. 5). Most strikingly, plasma insulin concentrations at week 22 were markedly lower in hyperglycaemic mice that presented both diabetogenic alleles (Fig. 5, lower panel).

Modulation of the effects of Nidd/SJL and Nob1 on the prevalence of diabetes in the NZO $\times$ SZO backcross by dietary fat. It is well recognized that obesity and insulin resistance are enhanced by an increased fat content of the diet [9]. The susceptibility to the dietary fat is strain specific, presumably because it is conferred by a genetic predisposition. In order to elucidate whether the effects of the diabetes susceptibility loci Nidd/SJL and Nobl required a high fat diet, a population of backcross mice $(n=74)$ was raised on a standard diet in parallel with those receiving the high fat diet.

Figure 6 illustrates a comparison of the effects of the diabetogenic alleles at a standard and a high-fat diet. At week 22, the prevalence of diabetes was increased by the high fat diet to a small extent only (Fig. 6A). Furthermore, the effect of the diabetogenic allele Nidd/SJL was essentially identical in both groups at week 22. At earlier time points, however, the high-fat diet appeared to markedly enhance the effect of Nidd/SJL, resulting in a marked acceleration of the development of diabetes.

In order to further characterise the effect of Nidd/SJL in mice on the standard diet, the data were analysed with regard to the genotypes at both diabetes susceptibility loci Nidd/SJL and Nob1 (Fig. 6B). Carriers of Nidd/SJL exhibited a markedly higher prevalence of diabetes $(52.4 \%$ at week 22$)$ as compared with mice that lacked the diabetogenic allele $(10 \%)$, an effect that was similar to that observed on the high-fat diet (see Fig. 2B). Surprisingly, however, Nobl failed to enhance the effect of Nidd/SJL on the prevalence of diabetes in the mice on the standard diet (Fig. 6B). This lack of effect of Nobl became further evident, when the time course was analysed with regard to both genotypes at Nidd/SJL and Nob1 (Fig. 6C). In contrast to the results observed on the high-fat diet, Nob1 failed to accelerate the development of diabetes in mice on the standard diet. Thus, the accelerated development of diabetes in
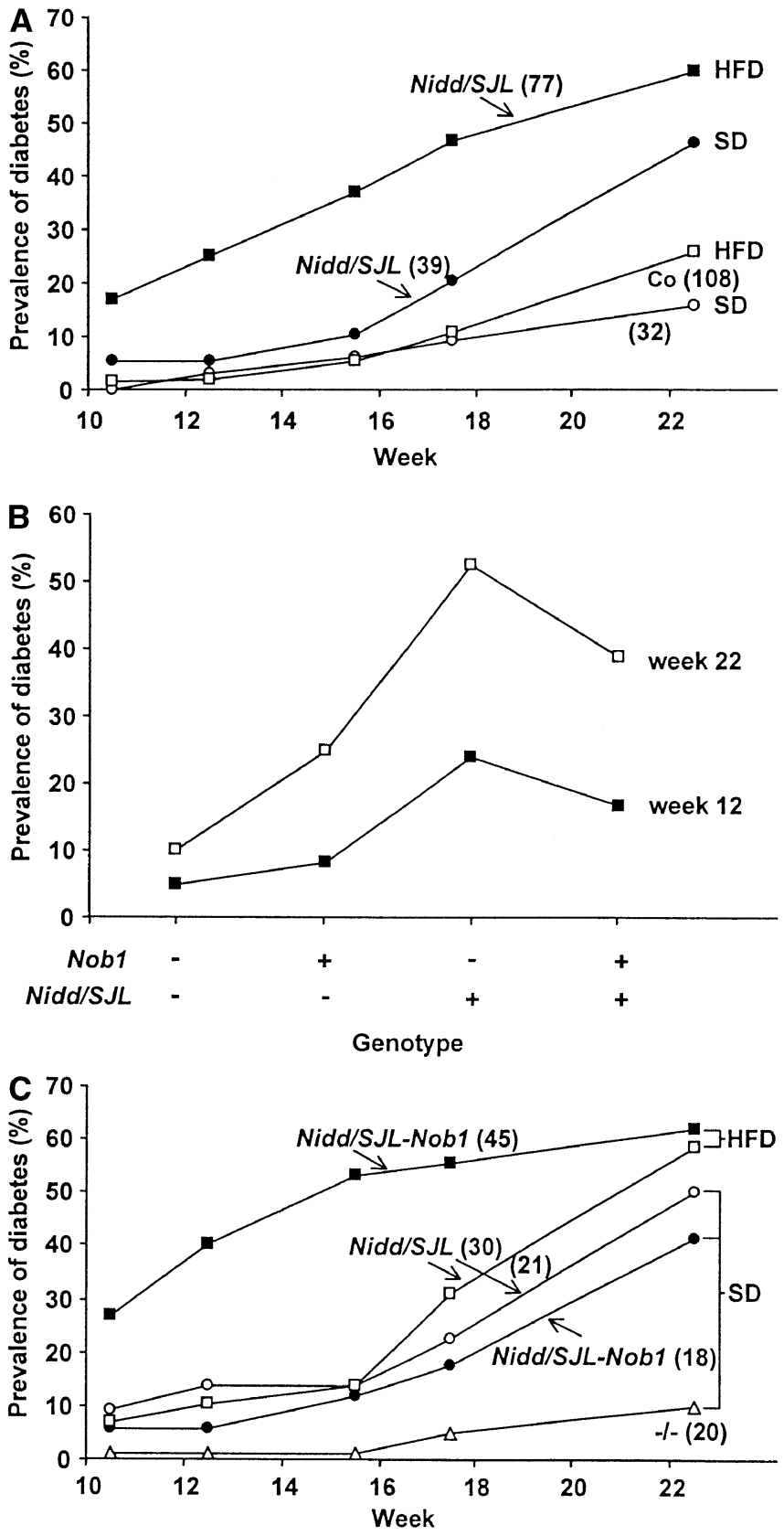

Fig. 6. Effects of Nidd/SJL and Nobl on the prevalence of diabetes in $\mathrm{NZO} \times \mathrm{F} 1(\mathrm{SJL} \times \mathrm{NZO})$ backcross mice at a standard as compared with a high-fat diet. A Time course of the development of diabetes at high-fat (HFD) or standard (SD) diet in the presence or absence of Nidd/SJL. The number of mice in each group are given in parenthesis. B Lack of interaction of Nidd/SJL and Nobl (homozygosity at D5Mit392) at the standard diet. Numbers of mice in each group: 20 (-/-), 12 (Nobl only), 21 (Nidd/SJL only),18 (Nidd/SJL-Nobl). C Time course of the development of diabetes as analysed for the presence of both (Nidd/SJL-Nobl) or only one (Nidd/SJL) diabetogenic allele

Nidd/SJL carriers on the high fat diet (Fig. 6A) reflects the enhancing action of Nobl. Consequently, the diabetogenic effect of Nobl, but not that of Nidd/SJL, appears to be dependent on the dietary fat content. 


\section{Discussion}

In the pathogenesis of rodent or human Type II diabetes, a period of hyperinsulinaemia, compensating for the obesity-associated insulin resistance, precedes overt hyperglycaemia. Glucose homeostasis decompensates, and hyperglycaemia begins, when the capacity of the beta cells falls below a critical threshold. Accordingly, the decompensation of glucose homeostasis in our NZO $\times$ SJL backcross model is a consequence of insulin resistance and subsequent beta cell failure. Progression of the disease to a Type II diabetes-like hyperglycaemia is preceded by obesity-associated insulin resistance, and requires genes conferring the susceptibility for islet cell failure, e.g. those located in the locus Nidd/SJL. These data further confirm the validity of the concept that Type II diabetes in rodents ('diabesity') is produced by the combination of obesity and diabetes genes [6,10, 11, 12], and that it closely resembles the human disease.

Obesity-induced diabetes has previously been studied in several other mouse models including strains with the monogenic $o b / o b$ and $d b / d b$ mutations [1]. Here we have chosen the NZO mouse model because of its polygenic pathogenesis which seems to be similar to that of the human metabolic syndrome. Most importantly, the strong association of body weight with the risk of Type II diabetes are common features of the mouse model and the human disease. Furthermore, the polygenic model offers the possibility to find novel interactions between genes, or interactions between genes and dietary factors, and to ultimately identify the molecular basis of these interactions.

The mechanism of beta-cell destruction in obese mouse models, and also in the human disease, is entirely unknown. Based on the present data, two different, at present entirely speculative scenarios might be discussed. Firstly, it has previously been observed that obesity-associated insulin resistance, e.g. in the $d b / d b$ mouse, produces a marked islet hyperplasia [13]. Similarly, islet hyperplasia was also observed in NZO mice, with approximately $90 \%$ of islet tissue consisting of beta cells [1]. Therefore, it might be speculated that in mice carrying diabetes genes, the proliferative stimulus which normally induces islet hyperplasia could cause apoptosis. Secondly, beta-cell destruction could be produced by the effects of fatty acids ('lipotoxicity'), as suggested previously $[14,15]$. Our data reveal that the development of diabetes in carriers of the diabetogenic alleles Nob1 and Nidd/SJL is markedly accelerated by a high fat diet, supporting the concept of 'lipotoxicity', and indicating that the combination of Nobl and Nidd/SJL confers a higher sensitivity to the deleterious effects of fatty acids or of lipid accumulation on pancreatic islets.

Our data exclude the possibility that Nidd/SJL increases body weight or aggravates insulin resistance, thereby contributing to the diabetogenic effect of other obesity genes and indirectly producing islet cell failure. Thus, it seems likely that the gene(s) responsible for the diabetogenic effect of Nidd/SJL play(s) a role in islet cell function and produce(s) islet cell failure directly. Highly significant LOD scores were obtained over a range of about $20 \mathrm{cM}$, suggesting that the locus harbours more than one diabetogenic gene. This region of the mouse genome is syntenic with the human chromosome 1 and contains approximately 300 known genes or transcripts. So far, however, we have not been able to identify a candidate gene involved in islet cell function in this region.

Nidd/SJL was mapped to a chromosomal region in which other diabetes genes have been identified previously. A diabetogenic allele on the proximal part of chromosome 4 (Niddl) was identified in the NON strain [3]. Maximum LOD scores for Niddl were obtained with a single nucleotide polymorphism [16] within the coding region of the Lepr gene. For the diabetogenic locus described here, maximum LOD scores for blood glucose were found 8-28 cM distal of Lepr. Thus, it appears unlikely that Nidd/SJL comprises the same variant gene as Nidd1. A second known diabetogenic locus on chromosome 4, Idd11, has been identified in NOD mice and was mapped close to the $\mathrm{Na}+\mathrm{H}+$ exchanger [17] which is located $7.6 \mathrm{cM}$ distal from D4Mit278. Thus, the possibility cannot be excluded that the gene(s) responsible for the effects of Nidd/SJL and IddIII are identical. It should be noted that the NOD, NON, and SJL strains are derived from Swiss outbred colonies (Mouse Genome Informatics database, The Jackson Laboratory). NOD was separated at F6 from the ICR/Swiss-derived CTS-strain. NON was separated from NOD at F13. Furthermore, SJL originated from a Swiss Webster outbred stock brought to the Jackson Laboratory between 1938-1943. It is therefore conceivable that NOD, NON, and SJL carry the same diabetogenic genes.

The diabetogenic effect of Nidd/SJL was markedly accelerated and aggravated by the obesity and insulin resistance QTL Nob1. Together, Nidd/SJL and Nob1 accounted for about $90 \%$ of the prevalence of diabetes in the backcross population. This finding is surprising because Nobl was responsible for only a small portion of the trait obesity. More importantly, the enhancing effect of Nobl was dependent on a high-fat diet. Thus, we speculate that Nobl is involved in storage or metabolism of lipids. The diabetogenic allele produces insulin resistance and, when exposed to excess exogenous lipids, exerts deleterious effects on the beta cell.

Acknowledgements. The authors are indebted to S. Winandy and C. Müller for skilful technical assistance.

\section{References}

1. Herberg L, Coleman DL (1977) Laboratory animals exhibiting obesity and diabetes syndromes. Metabolism 26:59-98

2. Ortlepp JR, Kluge R, Giesen K et al. (2000) A Metabolic Syndrome of Hypertension, Hyperinsulinaemia, and Hy- 
percholesterolemia in the New Zealand Obese (NZO) Mouse. Eur J Clin Invest 30:195-202

3. Leiter EH, Reifsnyder PC, Flurkey K, Partke HJ, Junger E, Herberg L (1998) NIDDM genes in mice. Deleterious synergism by both parental genomes contributes to diabetic thresholds. Diabetes 47:1287-1295

4. Plum L, Kluge R, Giesen K, Altmüller J, Ortlepp JR, Joost H-G (2000) Type-2-diabetes-like hyperglycaemia in a backcross model of New Zealand obese (NZO) and SJL mice: Characterization of a susceptibility locus on chromosome 4 and its relation with obesity. Diabetes 49:1590-1596

5. Kluge R, Giesen K, Bahrenberg G, Plum L, Ortlepp JR, Joost H-G (2000) Two quantitative trait loci for obesity and insulin resistance (Nob1, Nob2) and their interaction with the leptin receptor locus (Lepr $\left.{ }^{A 720 T / T 1044 I}\right)$ in New Zealand obese (NZO) mice. Diabetologia 43:1565-1573

6. Leiter EH, Herberg L (1997) The polygenetics of diabesity in mice. Diabetes Rev 5:131-148

7. Lincoln S, Daly M, Lander E (1992) Mapping genes controlling quantitative traits with Mapmaker/QTL 1.1. 2nd edn. Whitehead Institute Technical Report

8. Lincoln S, Daly M, Lander E (1992) Constructing genetic maps with Mapmaker/EXP 3.0. 3rd edn. Whitehead Institute Technical Report

9. Surwit RS, Kuhn CM, Cochrane C, McCubbin JA, Feinglos MN (1988) Diet induced type II diabetes in C57BL/6 J mice. Diabetes 37:1163-1167
10. Shafrir E (1992) Animal models of non-insulin-dependent diabetes. Diabetes Metab Rev 8:179-208

11. Leiter EH (1993) Obesity genes and diabetes induction in the mouse. Crit Rev Food Sci Nutr 33:333-338

12. Shafrir E (1996) Development and consequences of insulin resistance: lessons from animals with hyperinsulinaemia. Diabetes Metab 22:122-131

13. Gapp DA, Leiter EH, Coleman DL, Schwizer RW (1983) Temporal changes in pancreatic islet composition in C57BL/6J-db/db (diabetes) mice. Diabetologia 25:439443

14. Unger RH (1995) Lipotoxicity in the pathogenesis of obesity-dependent NIDDM. Genetic and clinical implications. Diabetes 44:863-870

15. Kakuma T, Lee Y, Higa M et al. (2000) Leptin, troglitazone, and the expression of sterol regulatory element binding proteins in liver and pancreatic islets. Proc Natl Acad Sci USA 97:8536-8541

16. Igel M, Becker W, Herberg L, Joost HG (1997) Hyperleptinemia, leptin resistance and polymorphic leptin receptor in the New Zealand Obese (NZO) mouse. Endocrinology 138:4234-4239

17. Morahan G, McClive P, Huang D, Little P, Baxter A (1994) Genetic and physiological association of diabetes susceptibility with raised $\mathrm{Na}+\mathrm{H}+$ exchange activity. Proc Natl Acad Sci USA 91:5898-5902 